\title{
Chiral Spin Liquid in a Frustrated Anisotropic Kagome Heisenberg Model
}

\author{
Yin-Chen He, ${ }^{1}$ D. N. Sheng, ${ }^{2}$ and Yan Chen ${ }^{1,3}$ \\ ${ }^{1}$ Department of Physics, State Key Laboratory of Surface Physics and Laboratory of Advanced Materials, \\ Fudan University, Shanghai 200433, China \\ ${ }^{2}$ Department of Physics and Astronomy, California State University, Northridge, California 91330, USA \\ ${ }^{3}$ Department of Physics and Center of Theoretical and Computational Physics, \\ The University of Hong Kong, Pokfulam Road, Hong Kong, China \\ (Received 10 January 2014; revised manuscript received 20 February 2014; published 4 April 2014)
}

\begin{abstract}
Kalmeyer-Laughlin (KL) chiral spin liquid (CSL) is a type of quantum spin liquid without time-reversal symmetry, and it is considered as the parent state of an exotic type of superconductor-anyon superconductor. Such an exotic state has been sought for more than twenty years; however, it remains unclear whether it can exist in a realistic system where time-reversal symmetry is breaking ( $T$ breaking) spontaneously. By using the density matrix renormalization group, we show that KL CSL exists in a frustrated anisotropic kagome Heisenberg model, which has spontaneous $T$ breaking. We find that our model has two topological degenerate ground states, which exhibit nonvanishing scalar chirality order and are protected by finite excitation gap. Furthermore, we identify this state as KL CSL by the characteristic edge conformal field theory from the entanglement spectrum and the quasiparticles braiding statistics extracted from the modular matrix. We also study how this CSL phase evolves as the system approaches the nearest-neighbor kagome Heisenberg model.
\end{abstract}

DOI: 10.1103/PhysRevLett.112.137202

PACS numbers: 75.10.Kt, 05.30.Pr, 75.10.Jm, 75.40.Mg

Topological order, an exotic state of matter that hosts fractionalized quasiparticles with anyonic braiding statistics, is one of the core topics in modern condensedmatter physics [1]. A quantum spin liquid (QSL) [2] is a prominent example of topological order, which is thought to exist in some frustrated magnets [3]. Among various types of QSL [3-11], there is a class of time-reversal symmetry violating QSL called chiral spin liquid (CSL) [12-14]. A CSL shares some similar properties with the fractional quantum Hall effect; however, the CSL is special for both possessing topological order and spontaneous time-reversal symmetry breaking.

The simplest CSL is the Kalmeyer-Laughlin (KL) CSL ( $\nu=1 / 2$ Laughlin state) [12], in which spinons obey semionic fractional statistics. It is theoretically shown that if one dopes the KL CSL with holes [15], an exotic type of superconductivity-anyon superconductivity [16] — will emerge. Inspired by the fundamental interest and prospect of finding exotic superconductors, researchers have shown much interest in the KL CSL [17-29]. There was no experimental or theoretical evidence supporting the existence of this state until very recently. Several artificial models were found that can host a KL state [26-28]. For example, one can directly induce scalar chirality order by a 3 -spin parity and time-reversal-violating interaction [28] on a kagome lattice to produce the KL state. However, it remains elusive whether the KL state can exist in a system with time-reversal symmetry, which may be more closely related to real materials. It has been suggested that the
KL state may exist in magnetic frustrated systems through spontaneously breaking time-reversal symmetry $[12,17]$, which are among the most difficult systems for theorists to study exactly.

In this Letter, we show that the KL state is the ground state of a frustrated anisotropic kagome Heisenberg model (KHM) by using the density matrix renormalization group (DMRG) [30], a numerical method which has been proven powerful in solving quasi-one-dimensional frustrated systems [31-34]. Compared with the previous systems with multiple spin interactions [26-28], the system we study here only involves two spin interactions, and the Hamiltonian has time-reversal symmetry. By the technique developed in Refs. [35,36], we find two topologically degenerate ground states, both of which break time-reversal symmetry spontaneously and exhibit a nonvanishing scalar chirality order. We also get a finite energy excitation gap and small correlation length, which support that we have a gapped phase. Furthermore, the entanglement spectrum of the ground states fits the edge conformal field theory of the KL state. Last but not least, we calculate the modular matrix using the two ground states [36-38], which gives the braiding statistics [1] of emergent anyons that is the same as what is expected for the KL state. To the best of our knowledge, this is the first model that breaks timereversal symmetry spontaneously and hosts a KL CSL. We also show how the system evolves as it approaches the nearest-neighbor KHM.

Model Hamiltonian.-We study a frustrated anisotropic KHM, whose Hamiltonian is 


$$
H=J \sum_{\langle i, j\rangle} S_{i} \cdot S_{j}+J_{2} \sum_{\langle\langle i, j\rangle\rangle} S_{i}^{z} S_{j}^{z}+J_{3} \sum_{\langle\langle\langle i, j\rangle\rangle\rangle} S_{i}^{z} S_{j}^{z},
$$

where the nearest-neighbor interaction $\langle i, j\rangle$ is the isotropic Heisenberg interaction, and the second $\langle\langle i, j\rangle\rangle$ and third $\langle\langle\langle i, j\rangle\rangle\rangle$ nearest-neighbor interactions are Ising-type interactions with the same magnitude, $J_{2}=J_{3}=J^{\prime}$. All the interactions are antiferromagnetic, and we take $J=1$. To establish the nature of the CSL here, we mainly focus on the point $J^{\prime}=1$, where we find the CSL is very robust.

We calculate the kagome lattice spin system wrapped on a cylinder with YC or XC geometry (see the Supplemental Material [39]). Both finite DMRG and infinite DMRG (iDMRG) [40] are used, and those two different algorithms give almost the same results with differences around the truncation error (energy $\sim 10^{-6}$, entropy $\sim 10^{-4}$ ) (see the Supplemental Material [39]). The CSL spontaneously breaks time-reversal symmetry; thus, we use both real and complex variable codes, although the Hamiltonian is real. We mainly focus on the system with an even number of sites on the column where the finite size effect is smaller. We have kept up to 8000 states in the DMRG simulation, and the truncation error is smaller than $10^{-6}$ for $\mathrm{YC} 8, \mathrm{XC} 8$ cylinders and $5 \times 10^{-5}$ for YC12, XC12 cylinders. These truncation errors are small enough to obtain the highly accurate results for the gapped CSL we find.

Degenerate ground states and their properties.Topological degeneracy is usually defined on a torus, but for an infinite cylinder (or sufficiently long cylinder), one can also have a full set of topological degenerate states in the bulk of the system. Using the technique developed in Ref. [35], we can get two topological degenerate ground states $\psi_{1}$ and $\psi_{s}$, which are distinguished by the absence or presence of a spinon line, as shown in Fig. 1(b). We obtain $\psi_{1}$ naturally in the conventional DMRG simulation and get $\psi_{s}$ by creating edge spinons (pin or remove one site). On the other hand, by inserting a $2 \pi$ flux in the cylinder, we find that $\psi_{1}\left(\psi_{s}\right)$ adiabatically evolves into $\psi_{s}\left(\psi_{1}\right)$ [Fig. 1(c)], which indicates that the $2 \pi$ flux insertion will pump a spinon from one edge to the other edge, a property for the $\nu=1 / 2$ fractional quantum Hall state. We also calculate the one column overlap [35] between states at different fluxes, as shown in Fig. 1(d). From the overlaps $F_{1}(\theta)=|\langle\psi(\theta) \mid \psi(\theta+\pi / 3)\rangle|$, we know the state evolves adiabatically as the flux is inserted. Nevertheless, $F_{2}(\theta)=$ $|\langle\psi(0) \mid \psi(\theta)\rangle|$ decreases as the inserted flux $\theta$ increases from 0 to $2 \pi$. In particular, $\psi_{1}$ and $\psi_{s}$ have a very small overlap $(f \sim 0.1)$, supporting the idea that they are distinct states. The one column overlap $(f)$ between two degenerate states can also give us the correlation length of spinon $\varepsilon_{s}=-1 / \log f \sim 0.4$ [35]. From the symmetry properties of the entanglement spectrum [35] (also see Fig. 3), we also find that $\psi_{1}$ and $\psi_{s}$ are different topological degenerate ground states related to the absence or presence of the spinon line. The energy and entropy of the two states are

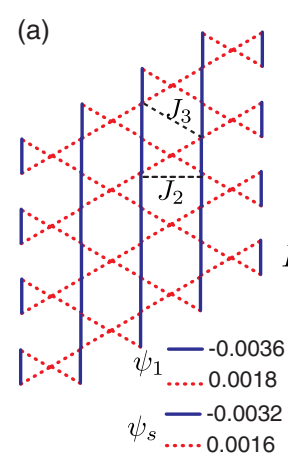

(b)
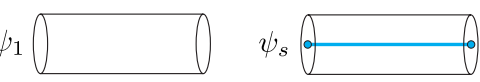

FIG. 1 (color online). (a) Illustration of YC8 $\left(L_{y}=4\right)$ kagome lattice. Here, we plot the bond spin correlation distribution of two ground states $\left\langle\boldsymbol{S}_{i} \cdot \boldsymbol{S}_{j}\right\rangle-e_{\alpha}$, where $e_{\alpha}$ is the average of the bond spin correlations, -0.206450 for $\psi_{1}$ and -0.206486 for $\psi_{s}$. (b) Two topological degenerate ground states $\psi_{1}$ and $\psi_{s}$ in CSL. Response of YC8 cylinder under flux insertion: (c) The energy evolution. (d) The one column overlap between states at different fluxes $F_{1}(\theta)=|\langle\psi(\theta) \mid \psi(\theta+\pi / 3)\rangle|$ and $F_{2}(\theta)=|\langle\psi(0) \mid \psi(\theta)\rangle|$. Here, $\psi(0)=\psi_{1}$ and $\psi(2 \pi)=\psi_{s}$.

very close (Table I), and their difference drops quickly as the system size increases. It is consistent with the theory of topological degeneracy, where the energy difference is expected to vanish exponentially with system width, $\Delta E \sim \exp \left(-L_{y} / \varepsilon_{s}\right)$.

To check whether the state spontaneously breaks time-reversal symmetry, we measure the scalar chirality order [17]

$$
\chi_{i}^{\triangleright(\triangleleft)}=\left\langle\boldsymbol{S}_{i_{1}} \cdot\left(\boldsymbol{S}_{i_{2}} \times \boldsymbol{S}_{i_{3}}\right)\right\rangle, \quad i_{1}, i_{2}, i_{3} \in \triangleright, \triangleleft .
$$

We find $\chi$ is homogeneous on a kagome lattice, where the up and down triangles have the same chirality $\chi^{\triangleright} \approx \chi^{\triangleleft}$. We also calculate the overlap between $\psi_{i}$ and its conjugate $\psi_{i}^{*}$, where a very small value $\left(3 \times 4 \times 16\right.$ YC8 cylinder $\sim 10^{-6}$, $3 \times 4 \times 24$ YC8 cylinder $\sim 10^{-8}$ ) is obtained. The orthogonality between $\psi_{i}$ and $\psi_{i}^{*}$ indicates that we have two orthogonal states with opposite chirality. We further compare these two states with the results of real variable code simulation, and we find $\psi_{i}$ and $\psi_{i}^{*}$ are minimal entangled states [the superposition states $\left(\psi \pm \psi_{i}^{*}\right) / \sqrt{2}$ will be maximal entangled states] of a spontaneous time-reversal symmetry-breaking system. The triplet gap and singlet gap are calculated on a finite cylinder embedded in the middle of the infinite cylinder (see the Supplemental Material [39]). From the correlation length, singlet and triplet gaps, we infer the existence of a large gap between the ground state and the excited states. To confirm the absence of magnetic order, we plot the spin correlation $\left\langle\boldsymbol{S}_{i} \cdot \boldsymbol{S}_{i+r}\right\rangle$ in Fig. 2(a), which clearly exhibits an exponentially decaying behavior. The nearest bond spin correlation is very homogeneous with a difference of around 1\% [Fig. 1(a)], so we can exclude the translational symmetry-breaking phase, 
TABLE I. The energy $E$, entropy $S$, correlation length $\xi$, chirality order $\chi \triangleright, \chi \triangleleft$, singlet gap $\Delta_{s}$, triplet gap $\Delta_{t}$. The energy and entropy of YC12 as well as XC12 cylinders have been extrapolated versus truncation error.

\begin{tabular}{lllccccr}
\hline \hline State & \multicolumn{1}{c}{$E$} & $S$ & $\xi$ & $\chi^{\triangleright}$ & $\chi^{\triangleleft}$ & $\Delta_{s}$ & $\Delta_{t}$ \\
\hline$\psi_{1}$, YC8 & -0.463771 & 2.880 & 1.08 & 0.09 & 0.09 & 0.17 & 0.40 \\
$\psi_{s}$, YC8 & -0.463756 & 2.875 & 0.85 & 0.09 & 0.09 & 0.16 & 0.40 \\
$\psi_{1}$, YC12 & -0.46356936 & 4.3688 & 0.60 & 0.104 & 0.104 & 0.24 & 0.42 \\
$\psi_{s}$, YC12 & -0.46356941 & 4.3687 & 0.60 & 0.104 & 0.104 & 0.24 & 0.42 \\
$\psi_{1}$, XC8 & -0.463630 & 2.711 & 0.79 & 0.095 & 0.095 & 0.14 & 0.35 \\
$\psi_{s}$, XC8 & -0.463643 & 2.719 & 0.87 & 0.093 & 0.093 & 0.15 & 0.35 \\
$\psi_{1}$, XC12 & -0.4635381 & 4.153 & 0.55 & 0.103 & 0.103 & 0.22 & 0.41 \\
$\psi_{s}$, XC12 & -0.4635382 & 4.154 & 0.55 & 0.103 & 0.103 & 0.22 & 0.41 \\
\hline \hline
\end{tabular}

such as a valence bond crystal. All these properties suggest a CSL phase.

As a self-consistency check, we also calculate properties of the system using a real-variable code. For a time-reversal symmetry-breaking phase, a real-variable code will yield a maximal entangled state $\tilde{\psi} \sim\left(\psi \pm \psi^{*}\right) / \sqrt{2} ; \tilde{\psi}$ has a doubly degenerate entanglement spectrum, and its entropy is $\ln 2$ larger than that of $\psi$. For the time-reversal symmetric state, $\tilde{\psi}$, the scalar chirality order in Eq. (2) is 0, but we can extract it from the chiral correlation function $\left\langle\chi_{i} \chi_{i+r}\right\rangle=$ $\left\langle\left[\boldsymbol{S}_{i_{1}} \cdot\left(\boldsymbol{S}_{i_{2}} \times \boldsymbol{S}_{i_{3}}\right)\right]\left[\boldsymbol{S}_{(i+r)_{1}} \cdot\left(\boldsymbol{S}_{(i+r)_{2}} \times \boldsymbol{S}_{(i+r)_{3}}\right)\right]\right\rangle$. Clearly observed in Fig. 2(b) is a long-range chiral correlation, $\sqrt{\lim _{r \rightarrow \infty}\left\langle\chi_{i} \chi_{i+r}\right\rangle} \approx 0.09$ (YC8) and 0.1 (YC12).

Entanglement spectrum.- The entanglement spectrum can serve as a fingerprint for a topological chiral phase [41]. In the DMRG simulation, one can naturally obtain the entanglement spectrum along a vertical cut. To see whether the entanglement spectrum fits the conformal field theory, one needs to calculate the momentum (along the $y$ direction) of each entanglement spectrum [36]. Since the system has a $U(1)$ symmetry, each spectrum has an $S_{z}$ quantum number. From Fig. 3, one can see the pattern of $\psi_{1}$ is symmetric about the positive and negative $S_{z}$, whereas that of $\psi_{s}$ is symmetric about $S_{z}=0$ and $S_{z}=1$. This distinct symmetry pattern is the consequence of the absence or presence of the spinon line as shown in Fig. 1(b). Moreover, one can clearly observe an entanglement
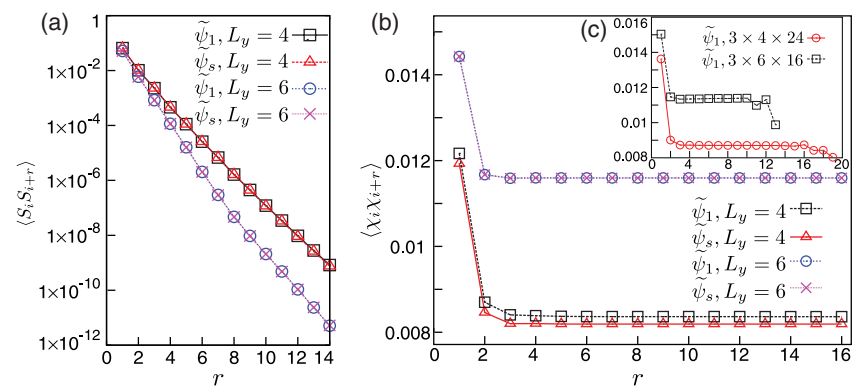

FIG. 2 (color online). (a) Spin correlation $\left\langle\boldsymbol{S}_{i} \cdot \boldsymbol{S}_{i+r}\right\rangle$ versus distance $r$. The chiral correlation $\left\langle\chi_{i} \chi_{i+r}\right\rangle$ versus the distance $r$ : (b) Results from the iDMRG. (c) Results from the finite DMRG. The system sizes we calculated are $3 \times 4 \times 24$ and $3 \times 6 \times 16 \mathrm{YC}$ cylinders. Here, $J^{\prime}=1$. spectrum gap and the degeneracy pattern $(\{1,1,2,3,5, \ldots\})$ of the low-lying spectra. This is in agreement with the edge conformal field theory of the KL state [36].

Braiding statistics from a modular matrix.-Next, we use $\psi_{1}$ and $\psi_{s}$ to calculate the modular matrix [1], $\mathcal{S}, \mathcal{U}$, which contains full information of a topological ordered phase. For a topological chiral state, the modular matrix is

$$
\mathcal{S}=\left(\begin{array}{ll}
S_{11} & S_{1 s} \\
S_{s 1} & S_{s s}
\end{array}\right), \quad \mathcal{U}=e^{-i(2 \pi / 24) c}\left(\begin{array}{cc}
h_{1} & 0 \\
0 & h_{s}
\end{array}\right)
$$

$S_{1 i}=S_{i 1}=d_{i} / D$, where $d_{i}$ is the quantum dimension of quasiparticle (type $i$ ) and $D=\sqrt{\sum_{i} d_{i}^{2}}$ is the total quantum dimension. The topological entanglement entropy [42,43] can also give the quantum dimension [44]. The entry $S_{i j}$ will give the braiding statistics of the anyon model. Furthermore, $c$ is the central charge of the system, and $h_{i}$ is called the topological spin, which determines the selfstatistics of the type- $i$ anyon. For Abelian anyons, one has $d_{i}=1$ and $S_{i j}=\exp \left(i \theta_{i j}\right) / \sqrt{D}$ where $\theta_{i j}$ is the phase coming from a type- $i$ anyon encircling a type- $j$ anyon.

Following the procedure outlined in Refs. [36,37], we get the modular matrix of the YC8 cylinder using Monte Carlo sampling [45]

$$
\mathcal{S}=\frac{1}{\sqrt{2}}\left(\begin{array}{cc}
1 & 1 \\
1 & -1
\end{array}\right)+\frac{10^{-2}}{\sqrt{2}}\left(\begin{array}{lc}
-0.42 & -2.2 \\
-1.26 & 0.76-0.15 i
\end{array}\right)
$$

and

$$
\mathcal{U}=e^{-i(2 \pi / 24)}\left(\begin{array}{ll}
1 & 0 \\
0 & i
\end{array}\right) \times\left(\begin{array}{cc}
e^{0.011 i} & 0 \\
0 & e^{-0.006 i}
\end{array}\right)
$$

Generally, from the modular matrices, one can know: (1) there are two types of quasiparticles, a trivial vacuum 1 and a spinon $s$ - they are all Abelian quasiparticles with quantum dimension $d_{i}=1$; (2) the spinon has semionic braiding statistics relative to itself; (3) the fusion rules are $1 \times 1=s \times s=1,1 \times s=s \times 1=s$; (3) the topological spins of the two quasiparticles are $h_{1}=1, h_{s}=i$; and (4) the central charge is $c=1$. From these results, we can conclude that this CSL state is the KL state. 
(a)

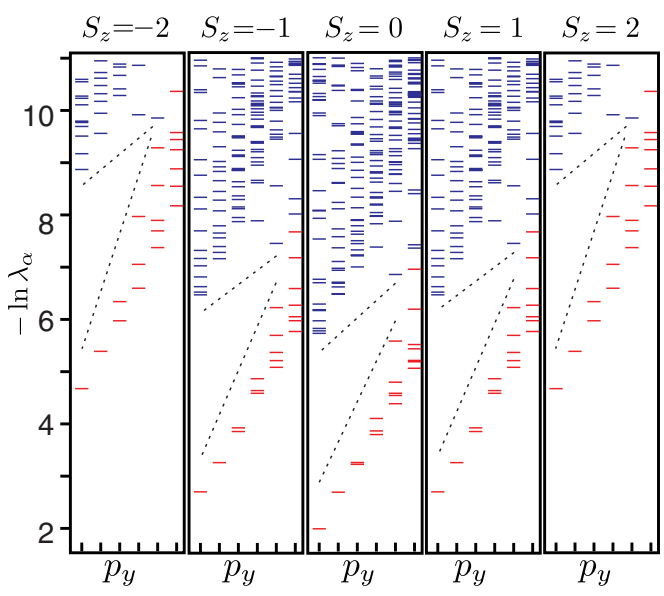

(b)

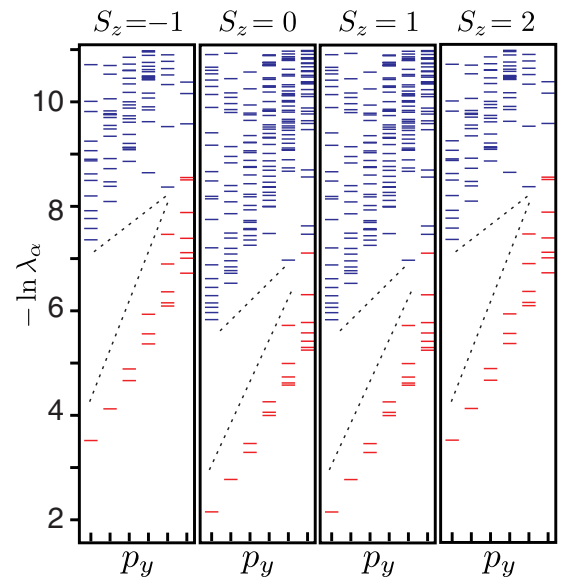

FIG. 3 (color online). Entanglement spectrum of YC12 cylinder $\left(L_{y}=6\right)$ (a) $\psi_{1}$ and (b) $\psi_{s}$. The horizontal axis is the momentum along the $y$ direction, $p_{y}=0,2 \pi / 6, \ldots, 5 \times 2 \pi / 6$ (up to a global shift). The leading spectra are marked in red, above which there is a spectrum gap marked with a dashed line. Other geometry and size samples have similar results.

It is interesting to know the mechanism for the KL CSL phase. An intriguing proposal for the emergence of a CSL on the kagome lattice is given in Ref. [14]. They found that a $J_{1} \sim J_{2} \sim J_{3} \mathrm{SU}(2)$ Heisenberg interaction on a kagome lattice will lead to a classical chiral state with 12 spins pointing toward the corners of a cuboctahedron. Quantum fluctuation will disrupt the classical order and result in a CSL state. However, our Ising antiferromagnetic interaction does not favor such a complex classical state, as it only prefers to align spins antiparallel along the $z$ direction. Moreover, the CSL proposed there is a $Z_{2}$ CSL, different from the KL-CSL we find here. We think the CSL here comes from quantum frustration on the kagome lattice as originally proposed by a KL [12], and the Ising antiferromagnetic interactions only play the role of stabilizing the CSL state through enhancing the repulsive interactions between spin particles. In a parallel work, a CSL has also been found in an SU(2) rotational invariant Heisenberg model [46] on a kagome lattice. It is interesting to study whether real material relevant interactions [47] may also

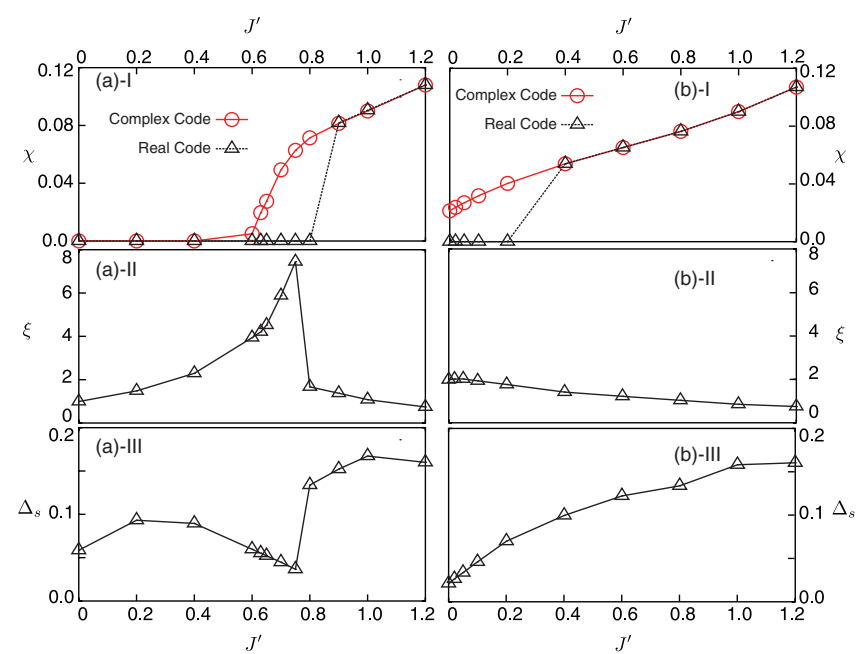

FIG. 4 (color online). $J^{\prime}$-dependent behavior of the two topological degenerate sectors: (a) normal sector $\psi_{1}$ and (b) spinon sector $\psi_{s}$. Here, we show results of calculations for the YC8 cylinder: (I) the chirality order, $\chi$ [Eq. (2)], where for the real code, we define $\chi=\sqrt{\lim _{r \rightarrow \infty}\left\langle\chi_{i} \chi_{i+r}\right\rangle}$; (II) the correlation length $\xi$ of the ground state; (III) the singlet gap $\Delta_{s}$ of the ground state.

stabilize the KL state on the kagome lattice, which will help to find a KL CSL or even anyon superconductor in real materials such as herbertsmithite $\mathrm{ZnCu}_{3}(\mathrm{OH})_{6} \mathrm{Cl}_{2}$.

Chiral spin liquid and nearest-neighbor KHM.-In the following, we study how the CSL evolves into the limit $\left(J^{\prime}=0\right)$, nearest-neighbor KHM, where a possible $Z_{2}$ SL was previously discovered [31]. To systematically study the state evolution, we use both real- and complex-variable codes, where we find the former has a bias towards a time-reversal invariant spin liquid (TSL) [48], whereas the latter has a bias towards a CSL (see Supplemental Material [39]). In the whole region $J^{\prime} \in[0,1.2]$, the two degenerate ground states $\psi_{1}$ and $\psi_{s}$ always exist; moreover, due to the finite size effect, these two states behave differently as $J^{\prime}$ decreases as shown in Fig. 4.

Because of the bias of the real and complex code, we find in some regions (transition region) two codes give different states: TSL and CSL. As shown by the scalar chirality order, the transition region for $\psi_{0}$ is around $(0.6,0.8)$ and that for $\psi_{s}$ is around $(0.0,0.4)$. The energy difference of TSL and CSL is very small $\left(\sim 10^{-5}\right)$, indicating the strong competition between these two states. We find that, for the $\psi_{0}$, TSL has a lower energy than CSL when $J^{\prime} \lesssim 0.7$; in contrast, for $\psi_{s}$, the CSL always has a slightly lowerenergy than TSL. The CSL in the spinon sector may be related to the excited state with nonzero Chern number on nearest-neighbor KHM found in the exact diagonalization study [49]. The two sectors may also behave similarly as the system size increases. The quantum phase transition between the two topological phases, CSL and TSL, may result in new intriguing critical behavior, which may explain the results we obtain in the 
transition region. We leave these open and challenging topics for future study.

Conclusion.-Using the DMRG, we numerically show that the KL CSL is the ground state of a frustrated anisotropic KHM. We provide various evidence to support the KL CSL's existence, especially the presence of finite scalar chirality order, topological degeneracy, large energy gap, and the verification of the semionic fractional statistics of the spinons. Our work shows that the KL CSL can emerge in a realistic model for the first time, which only involves time-reversal invariant two-spin exchange interaction terms. Furthermore, we study how the system evolves into the nearest-neighbor KHM and provide some explanation of the competing evidence between the CSL and $Z_{2}$ SL. It would be interesting to study much larger systems using different approaches (such as tensor network or projected wave function) to further establish the topological phase and exclude the possibility of a weak order.

D. N. S. thanks Shoushu Gong and Wei Zhu for extensive discussions. This work was supported by the State Key Programs of China (Grants No. 2012CB921604 and No. 2009CB929204), the National Natural Science Foundation of China (Grants No. 11074043 and No. 11274069) (Y.-C.H. and Y.C.), and the U.S. National Science Foundation under Grant No. DMR0906816 (D. N. S.).

[1] X.-G. Wen, Int. J. Mod. Phys. B 04, 239 (1990).

[2] P. W. Anderson, Mater. Res. Bull. 8, 153 (1973).

[3] L. Balents, Nature (London) 464, 199 (2010).

[4] N. Read and S. Sachdev, Phys. Rev. Lett. 66, 1773 (1991).

[5] D. S. Rokhsar and S. A. Kivelson, Phys. Rev. Lett. 61, 2376 (1988).

[6] T. Senthil and M. P. A. Fisher, Phys. Rev. B 62, 7850 (2000).

[7] R. Moessner and S. L. Sondhi, Phys. Rev. Lett. 86, 1881 (2001).

[8] L. Balents, M. P. A. Fisher, and S. M. Girvin, Phys. Rev. B 65, 224412 (2002).

[9] A. Kitaev, Ann. Phys. (Amsterdam) 303, 2 (2003).

[10] M. A. Levin and X.-G. Wen, Phys. Rev. B 71, 045110 (2005).

[11] Y. Ran, M. Hermele, P. A. Lee, and X.-G. Wen, Phys. Rev. Lett. 98, 117205 (2007).

[12] V. Kalmeyer and R. B. Laughlin, Phys. Rev. Lett. 59, 2095 (1987);V. Kalmeyer and R. B. Laughlin, Phys. Rev. B 39, 11879 (1989).

[13] H. Yao and S. A. Kivelson, Phys. Rev. Lett. 99, 247203 (2007).

[14] L. Messio, B. Bernu, and C. Lhuillier, Phys. Rev. Lett. 108, 207204 (2012).

[15] R. B. Laughlin, Phys. Rev. Lett. 60, 2677 (1988).

[16] F. Wilczek, Fractional Statistics and Anyon Superconductivity (World Scientific, Singapore, 1990).

[17] X.-G. Wen, F. Wilczek, and A. Zee, Phys. Rev. B 39, 11413 (1989).

[18] Y.-H. Chen, F. Wilczek, E. Witten, and B. I. Halperin, Int. J. Mod. Phys. B 03, 1001 (1989).
[19] K. Yang, L. K. Warman, and S. M. Girvin, Phys. Rev. Lett. 70, 2641 (1993).

[20] F. D. M. Haldane and Daniel P. Arovas, Phys. Rev. B 52, 4223 (1995).

[21] D.-H. Lee and C. L. Kane, Phys. Rev. Lett. 64, 1313 (1990).

[22] G. Baskaran, Phys. Rev. Lett. 63, 2524 (1989).

[23] J. Marston and C. Zeng, J. Appl. Phys. 69, 5962 (1991).

[24] Y.-F. Wang, Z.-C. Gu, C.-D. Gong, and D. N. Sheng, Phys. Rev. Lett. 107, 146803 (2011).

[25] M. Hermele, V. Gurarie, and A. M. Rey, Phys. Rev. Lett. 103, 135301 (2009).

[26] D. F. Schroeter, E. Kapit, R. Thomale, and M. Greiter, Phys. Rev. Lett. 99, 097202 (2007); R. Thomale, E. Kapit, D. F. Schroeter, and M. Greiter, Phys. Rev. B 80, 104406 (2009).

[27] A. E. B. Nielsen, J. I. Cirac, and G. Sierra, Phys. Rev. Lett. 108, 257206 (2012).

[28] B. Bauer, B. P. Keller, M. Dolfi, S. Trebst, and W. W. Ludwig, arXiv:1303.6963.

[29] M. Barkeshli, arXiv:1307.8194.

[30] S. R. White, Phys. Rev. Lett. 69, 2863 (1992); S. R. White, Phys. Rev. B 48, 10345 (1993).

[31] S. Yan, D. Huse, and S. R. White, Science 332, 1173 (2011).

[32] H.-C. Jiang, Z. Y. Weng, and D. N. Sheng, Phys. Rev. Lett. 101, 117203 (2008).

[33] H.-C. Jiang, Z. Wang, and L. Balents, Nat. Phys. 8, 902 (2012).

[34] S. Depenbrock, I. P. McCulloch, and U. Schollwöck, Phys. Rev. Lett. 109, 067201 (2012).

[35] Y.-C. He, D. N. Sheng, and Y. Chen, Phys. Rev. B 89, 075110 (2014).

[36] L. Cincio and G. Vidal, Phys. Rev. Lett. 110, 067208 (2013).

[37] Y. Zhang, T. Grover, A. Turner, M. Oshikawa, and A. Vishwanath, Phys. Rev. B 85, 235151 (2012).

[38] M. P. Zaletel, R. S. K. Mong, and F. Pollmann, Phys. Rev. Lett. 110, 236801 (2013).

[39] See the Supplemental Material at http://link.aps.org/ supplemental/10.1103/PhysRevLett.112.137202for cylinder geometry of kagome lattice, numerical algorithm.

[40] I. P. McCulloch, arXiv:0804.2509.

[41] H. Li and F. D. M. Haldane, Phys. Rev. Lett. 101, 010504 (2008).

[42] A. Kitaev and J. Preskill, Phys. Rev. Lett. 96, 110404 (2006).

[43] M. Levin and X.-G. Wen, Phys. Rev. Lett. 96, 110405 (2006).

[44] Because of the difficulty of extrapolating the converged entanglement entropy for larger systems (YC12, XC12) and the finite size effect, the topological entanglement entropy (TEE) extrapolated from our data has a deviation from the theoretical value $\log \sqrt{2}$. However, a near accurate TEE term is obtained for the CSL in the SU2 model [46] by keeping around 16000 states.

[45] A. W. Sandvik and G. Vidal, Phys. Rev. Lett. 99, 220602 (2007).

[46] S. S. Gong, W. Zhu, and D. N. Sheng, arXiv:1312.4519.

[47] H. O. Jeschke, F. Salvat-Pujol, and R. Valenti, Phys. Rev. B 88, 075106 (2013).

[48] A state from a real-variable code is always time-reversal symmetric. The time-reversal invariant state here means a state with an exponentially decaying chiral correlation instead of a long-range chiral correlation as seen in Fig. 2.

[49] C. Waldtmann, H.-U. Everts, B. Bernu, C. Lhuillier, P. Sindzingre, P. Lecheminant, and L. Pierre, Eur. Phys. J. B 2, 501 (1998). 